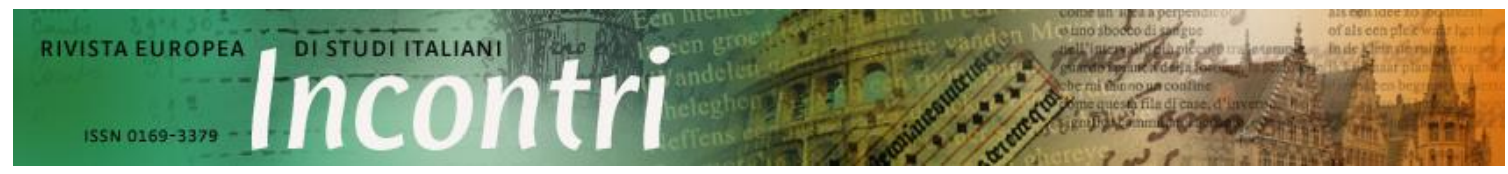

Anno 35, 2020 / Fascicolo 1 / p. 83-98 - www.rivista-incontri.nl - http://doi.org/10.18352/incontri.10336 (c) The author(s) - Content is licensed under a Creative Commons Attribution 3.0 Unported License Publisher: Werkgroepltalië Studies, supported by Utrecht University Library Open Access Journals

\title{
Italie obscure o Une terre pour les images? L'Italia artistico-letteraria di Bonnefoy e Orcel
}

\section{Novella Primo}

Pur appartenendo a generazioni diverse e considerando le profonde differenze di formazione e di poetica, sia Yves Bonnefoy (1923-2016) che Michel Orcel (nato nel 1952) costituiscono due esempi paradigmatici di interesse profondo e finemente competente verso la penisola italiana e la sua cultura, come emerge innanzitutto dalle rispettive produzioni saggistiche (con una maggiore curvatura di Bonnefoy verso la critica d'arte) e dalle loro traduzioni di autori italiani corredate da importanti riflessioni metatraduttive. Il comune interesse nei confronti dell'Italia è infatti corroborato dalla loro sapiente esperienza di traduttori di importanti autori italiani come Ariosto e soprattutto Leopardi; non ci si propone pertanto di comparare i due autori quanto di far "reagire" i loro testi, insieme colti e appassionati, per provare a profilare alcuni contorni dell'Italia artistico-letteraria vista d'oltralpe.

Il nostro discorso trarrà il suo abbrivio da Bonnefoy, di cui inizialmente verranno prese in considerazione le sue osservazioni, prevalentemente in direzione iconografica, a proposito del paesaggio italiano per poi soffermarsi sul versante letterario, con un cenno ad Ariosto e una focalizzazione del discorso tutta leopardiana. Analogamente, nel caso della produzione del secondo autore, da noi scelto per il suo percorso tra traduzione e critica accostabile a quello bonnefoyano, si accennerà alla particolare visione di un'Italie obscure, pressoché aniconica, con particolare attenzione al lavoro traduttorio ed ermeneutico compiuto sui Canti del Recanatese, da cui apparirà come il discorso su Leopardi diventi il trait d'union privilegiato tra $i$ due letterati francesi.

\section{L'Italia secondo Bonnefoy}

La civiltà artistica italiana ha costituito una delle principali fonti di ispirazione di Yves Bonnefoy, autorevole poeta francese contemporaneo che, tra i suoi modelli letterari, annovera poeti italiani quali Dante, Petrarca e soprattutto Leopardi. Altrettanto forte è l'interesse verso il paesaggio della nostra penisola, magistralmente espresso nel celeberrimo L'Arrière-pays, ${ }^{1}$ come si può evincere sin dalla premessa che l'autore scrive in occasione dell'uscita dell'edizione italiana del suo libro:

Dallo scoglio di Capraia al limitare crepuscolare delle strade verso Apecchio, o Camerino, dal mausoleo di Galla Placidia a Sant'Ivo alla Sapienza, o lungo le alte pareti di Orsanmichele, ma anche nel lieve sussurro dell'acqua sotterranea a San Clemente; e poi, fin dai primi giorni, alcuni sublimi versi di Dante - 'ma come i gru van cantando lor lai...' - fino ad altri, più

\footnotetext{
1 Y. Bonnefoy, L'Arrière-pays (1972), Paris, Gallimard, 2005; si cita da: Id., L'entroterra, ed. italiana a cura di Gabriella Caramore con un saggio introduttivo dell'autore, Roma, Donzelli, 2004.
} 
recentemente, non meno sconvolgenti, di Leopardi, l'Italia è stata per me, nella vita vissuta o in quella immaginata, tutto un labirinto di insidie e insieme di lezioni di sapienza, tutta una rete di segni di una misteriosa promessa. ${ }^{2}$

Bonnefoy associa indistintamente, in queste pagine incipitarie, le bellezze paesaggistiche dell'Italia a quelle artistiche, a loro volta rilette sempre grazie al filtro della memoria letteraria, mostrandosi alla ricerca di un 'vrai lieu' da ritrovare proprio nell'Italia centrale, tra Toscana, Umbria e Marche, individuati come luoghi elettivi per poter vivere con una maggiore pienezza rispetto ad altri contesti. Come sottolinea Gabriella Caramore, ${ }^{3}$ non è un caso che tale scelta ricada proprio sull'Italia e non sulle terre d'Oriente, da sogno per un uomo occidentale, ma anche troppo lontane, né sui pur amati luoghi della Grecia dove, secondo il poeta francese, i toni duri e dolorosi della tragedia non sono addolciti neanche dal clima; nel cuore della latinità, invece, Bonnefoy ritrova un luogo dove 'le asperità sono mitigate dalla dolcezza', ${ }^{4}$ dove le forme antiche si aprono alla modernità della storia, in cui riconoscere e ritrovare epifanicamente insomma una rasserenante coincidentia oppositorum. È soprattutto l'Italia del Quattrocento, e in genere quella rinascimentale delle regioni centrali, a orientare lo sguardo di Bonnefoy in direzione di un recupero della dimensione più autentica dell'esistenza umana perché nella pittura italiana di quel periodo, ad esempio grazie a Piero della Francesca, l'humanitas trova una nuova e sorgiva formulazione, mai proposta prima di allora, e saldamente radicata nel mirabile paesaggio circostante, come ben spiega Caramore:

Un umanesimo così in contraddizione, in cui l'umanità è esaltata e svilita al tempo stesso, non può che aver luogo in un preciso paesaggio fisico, in una geografia non coerente, con una vegetazione ricca e uniforme, che conosce sia il riverbero dell'esuberanza mediterranea sia il pallore lacustre delle regioni nordiche. Gli ulivi della Toscana, le brune colline delle Marche, l'Umbria che ha il colore dell'acqua sono come un fondale chiaroscurato e cangiante, dalle atmosfere dolci e ombrate che accoglie un pensiero dell'uomo tragico, certo, ma in cui la tragicità è appena deviata - alleviata forse, o addirittura consolata - dal senso ineluttabile di appartenere a un destino. ${ }^{5}$

Anche in altri suoi scritti, ad esempio nel commento alla traduzione italiana dei suoi Récits en rêve, ${ }^{6}$ Bonnefoy ha ammesso di essere felice, ma anche turbato nel vedere $i$ suoi racconti pubblicati in Italia in quanto 'l'Italia è il luogo dove queste immaginazioni hanno preso forma'. ${ }^{7}$ All'effetto di straniamento più generale cui potrebbe condurre ogni lavoro di traduzione, si aggiunge in questo caso una forma suppletiva di spaesamento dovuta al fatto che la versione sia in italiano per cui le sensazioni dello scrittore francese sembrano riconducibili a quelle descritte da Freud a proposito del perturbante, in quanto l'Italia rappresenta per lui una dimensione originaria e originale dell'esperienza:

l'Italia mi ha molto aiutato a ricordare come sotto le rappresentazioni, che fanno velo, vi sia una traccia, un presentimento dell'Unità. Quando vidi sorgere di fronte a me, in una

\footnotetext{
2 Id., Avant propos 2004, a Id., L'entroterra, cit., p. 5. Si segnala che il presente contributo rielabora, in alcune parti i capitoli 3 e 4 del volume: Novella Primo, 'Al chiaror delle nevi'. Poeti-traduttori francesi di Giacomo Leopardi a confronto, Lecce, Milella, 2012.

${ }^{3} \mathrm{G}$. Caramore, Introduzione a Bonnefoy, L'entroterra, cit., pp. XIV-XVII.

${ }^{4}$ Ivi, p. Xv.

${ }^{5}$ Ivi, pp. XVI-XVII.

${ }^{6}$ Y. Bonnefoy, Récits en rêve, Milano, Egea, 1992.

7 Lo ricorda Cesare Greppi nel suo 'L'autore spaesato', in: Semicerchio, XxX-XXXI (2004), p. 45.
} 
indimenticabile prima sera fiorentina, la sconvolgente facciata di Santa Maria Novella, e poi la massa chiusa ma silenziosamente respirante di Orsanmichele. ${ }^{8}$

E poi la scoperta dei luoghi italiani prosegue lungo le città più piccole e le campagne, dove l'autore è colpito dal semplice, dall'immediato che in esse si percepisce, riportandolo alla sua stessa infanzia. Lo stesso viaggio è infatti vissuto come un ritorno all'origine che permette a Bonnefoy, in modo apparentemente tortuoso, di rivivere, attraverso la traduzione italiana, il suo rapporto originario con la Francia.

Il motivo dell'unità da ricercare in Italia affiora anche nello scritto Une terre pour les images, pubblicato e tradotto per i tipi di Donzelli con il titolo La civiltà delle immagini in cui sono riuniti efficacemente saggi di storia dell'arte (Piero della Francesca, Andrea Mantegna, Tiepolo, Veronese...) e altri di letteratura italiana (Ariosto, poeti dell'Arcadia, Leopardi):

Esiste un'unità di questa [della civiltà italiana, N.d.A.], a dispetto di tutto ciò che separa o perfino contrappone, prendiamo questi nomi a caso, un Botticelli e un Magnasco, oppure Palladio e Bernini? E se ne esiste una, in cosa consiste questa unità, cosa la distingue da altre ricerche condotte in quegli stessi secoli in altre regioni d'Europa? È la questione che vorrei affrontare, dopo avere tentato di comprendere spiriti tanto differenti, per non dire antagonisti, quali Mantegna, Tiepolo, Ariosto, Leopardi.

Una questione simile ha molto senso, ai miei occhi, e posso anche dire che l'ho sempre avuta in mente fin dai primi giorni del mio interesse per l'Italia: essa sarà stata in ogni momento il punto di fuga di ogni mia prospettiva, e la risposta che ho creduto di poterle dare è anch'essa, nella mia riflessione, piuttosto antica, benché mi sia occorso del tempo per capire in modo sufficientemente chiaro, e stavolta servendomi di qualche concetto, ciò che all'inizio presentivo solo intuitivamente.

$\mathrm{Mi}$ è occorso del tempo, in quanto questa mia idea non è così semplice. Essa crede di vedere, in effetti, una dualità al cuore stesso di un'unità: la 'doppia postulazione'. ${ }^{9}$

Bonnefoy porta cioè avanti la tesi, già espressa da vari critici d'arte come Henri Focillon, del dualismo insito nell'arte italiana secondo cui esisterebbero due Italie:

una 'monumentale' e l'altra 'narrativa', una innamorata di un'arte dei numeri, di bellezza nelle proporzioni, del silenzio che nasce dalla contemplazione dell'Intellegibile, e l'altra interamente votata alle delizie della finzione, questa a lungo nutrita di racconti di miracoli o di martiri. (...) Ma come non vedere che non è tra le opere compiute, siano esse grande architettura o graziosa serie d'immagini, che occorre cercare le varie opposizioni, ma nel pensiero dei loro ideatori, nel desiderio che in profondità le anima? ${ }^{10}$

Bonnefoy oppone alla tensione superficiale tra monumentale e narrativo una dualità più profonda che si esprime spesso occultamente entro lo stesso artista la cui opera germinerà appunto da questo travaglio, espressione anche di un'opposizione tra finitudine e infinito. Attraverso la costruzione di immagini gli artisti di fatto costruiscono altrettanti mondi, aderendo alla verità della finitezza, si eleveranno all' 'armonia di una forma': 'l'Italia è in modo quasi innato una terra per le immagini'. ${ }^{11}$

Dalle speculazioni dell'intellettuale di Tours si profila, al di là di vieti stereotipi, un paesaggio che riflette un desiderio dell'essere in grado di incarnarsi nel bello formale, nella capacità di agganciare l'infinito al contingente, oltre il tempo e lo spazio:

\footnotetext{
${ }^{8}$ Ibidem.

${ }^{9}$ Y. Bonnefoy, La civiltà delle immagini. Pittori e poeti d'Italia, Roma, Donzelli, 2005, p. 4.

${ }^{10}$ Ivi, pp. 4-5.

11 Ivi, p. 9.
} 
Il desiderio d'essere [...] invece di potere andare dritto alla sua intuizione più vera, che sarebbe, diciamo, vedere l'infinito nella pozza d'acqua che evapora, o quella foglia che cade, o quella mano che stringe quell'altra, in un momento di una vita, deve passare per la seduzione di questa musica delle forme, di questa bellezza che denigra la finitudine, che scredita il tempo. ${ }^{12}$

Questo dibattito interiore tra due opposizioni è da Bonnefoy opportunamente storicizzato e contestualizzato entro la formazione cristiana e il conseguente radicamento dell'idea dell'incarnazione dei pittori e degli architetti italiani vissuti tra il Medioevo e il Barocco, non altrettanto ben conosciuta, a detta dell'autore francese, dagli artisti 'del Nord'. Secondo il poeta di Tours un'altra peculiarità dell'arte italiana risiederebbe nell'enfasi posta sulla vita interiore dell'artista per cui l'opera si propone di significare l'essere attraverso l'esposizione della creazione in una continua tensione tra 'ontologia della forma e pensiero della finitudine, tra sogno e incarnazione'. ${ }^{13}$ Per Bonnefoy, la critica permette di capire meglio se stesso e l'altro e di stabilire il primato della parole sulla langue, della poesia sul 'poème', proponendo una poetica della 'finitudine e della speranza' intesa come categoria metafisica e critica, di cui la compassione e il desiderio d'essere costituiscono i cardini imprescindibili. ${ }^{14}$

Fabio Scotto,$^{15}$ traduttore e studioso dell'opera di Bonnefoy, considera come dei veri e propri scritti fondativi i saggi Le tombe di Ravenna e L'atto e il luogo della poesia. Nel primo di essi si contrappone l'inganno concettuale alla verità della morte che stabilisce il suo vrai lieu nella finitudine e nella presenza nel mondo sensibile, laddove il saggio L'atto e il luogo della poesia pone l'accento sul valore soteriologico dell'atto poetico che reca in sé un messaggio di speranza, sia pur all'interno di una 'teologia negativa', in cui non resta che accettare il caso, ma anche la 'verità di parola', venendo meno la presenza degli dei.

Pagine esemplari del volume La civiltà delle immagini sono quelle dedicate ad Ariosto nel contributo Orlando, ma anche Angelica: all'interno del 'grande racconto' del Furioso e, pedinando le 'tracce dei desideri' dei personaggi ariosteschi, non sfugge a Bonnefoy la particolare valenza assunta dal paesaggio che da mero sfondo narrativo si semantizza. Lo scrittore francese si interroga sul perché Angelica iscriva 'compulsivamente' il nome suo e di Medoro sugli alberi. Cosa c'è insomma dietro la 'pulsione scritturale di Angelica - che è, notiamolo, un atto della parola, portato proprio là dove pullulano i tratti più propri della realtà naturale, antecedente il linguaggio - fosse di nuovo questione del giardino dell'Eden?" ${ }^{16}$ Lo scarto sarebbe dato proprio dal nome proprio che con la sua fissazione sulle cortecce arboree, permetterebbe un incontro tra persona e natura. Esse, pur non arrivando mai a mescolarsi totalmente, simili in questo all'olio e all'acqua, esprimono la speranza che l'essere naturale e mentale, grazie alla mediazione del segno grafico, possano riunificarsi.

Anche Michel Orcel si sofferma su quest'episodio, ${ }^{17}$ sottolineando come lo spazio edenico del locus amoenus sarà distrutto dal furor di Orlando, originato dalla Spaltung dell'eroe eponimo, che manifesterà una dissociazione psichica proprio in seguito alla decifrazione delle particolareggiate scritte sugli alberi di Angelica e Medoro.

\footnotetext{
12 Ibidem.

13 Ivi, p. 11.

14 P. Née, De la critique poétique selon Yves Bonnefoy, in: Littérature, 150 (juin 2008), pp. 85-124: p. 122.

${ }^{15} \mathrm{~F}$. Scotto, commento a Yves Bonnefoy, L'Opera poetica, a cura e con un saggio introduttivo di F. Scotto, traduzioni poetiche di D. Grange Fiori e F. Scotto, Milano, Mondadori (“I Meridiani”), 2010, p. 1616.

16 Ivi, p. 94.

17 M. Orcel, Italie obscure, Paris, Belin, 2001, p. 15.
} 


\section{Bonnefoy in ascolto della parola leopardiana}

Oltre Ariosto, un altro fondamentale ambito di confronto tra Bonnefoy e Orcel è costituito dalla traduzione e dallo studio approfondito dell'opera di Giacomo Leopardi. Nello scritto di Bonnefoy L'enseignement et l'exemple de Leopardi, la lucida consapevolezza del néant si manifesta in una scrittura in grado di rendere presente, attraverso la parola, la melodia, il mondo sensibile che determina una coscienza di sé da cui paradossalmente si genera un messaggio di speranza; si tratta pertanto di uno dei contributi più significativi che Bonnefoy ha dedicato allo scrittore di Recanati, occasionato da una celebrazione leopardiana nella sua terra natale.

Dopo gli asteismi di rito e l'individuazione nelle Marche di uno dei poli della sua ispirazione poetica, l'autore rivendica innanzitutto il valore didattico, di enseignement appunto, per l'Italia e per il mondo, della scrittura del Recanatese, formulando in modo molto chiaro il concetto dell'importanza della sua ricezione capillare, in particolare in Francia:

Et cela, non parce que j'ai la prétention de vous apprendre quoi que ce soit sur l'auteur des Canti et du Zibaldone, mais pour vous dire par quel chemin quelqu'un qui se préoccupe de la création poétique en France peut trouver à cette œuvre une signification tout à fait actuelle et même un encouragement et une aide. En d'autres mots, il s'agira pour moi de la réception, comme on dit, de Leopardi et de l'apport qu'il me paraît faire à la conscience de soi de la poésie. ${ }^{18}$

Il discorso rivolto specificamente a Leopardi è preceduto da un denso excursus sulla storia della poesia prima del Romanticismo, giustificata dal fatto che, se è vero che il poeta di Silvia fu sicuramente concentrato su di sé, ebbe tuttavia 'une visée d'emblée universelle et qui fut donc à l'écoute des pensées qui vers 1815 agitaient l'Europe'. ${ }^{19}$

Il richiamo al background storico di quegli anni vuole mettere in luce soprattutto quei cambiamenti legati alla percezione del mondo correlati al 'travail de la poésie' ${ }^{20}$ Un mutamento nella percezione della realtà empirica si ha, infatti, con l'Illuminismo quando il pensiero tende a rendersi autonomo rispetto alla precedente visione occidentale, dominata dalla fede cristiana, secondo cui il mondo era decifrato come un testo nel quale Dio si esprimeva con analogie e corrispondenze, riflesso della realtà trascendentale. Già con Galilei al discorso teologico subentra quello governato dalle leggi della natura e, ancora, con Rousseau al divino vengono riservate altre forme di espressioni, secondo un 'nouvel emploi de l'instrument symbolique, associé à une nouvelle idée du divin'. ${ }^{21}$ Il divino si manifesta secondo altre modalità, ma è percepibile nella meraviglia di un'alta vetta, di un paesaggio e altri eventi della natura. Bonnefoy parla, a questo riguardo, di una 'existentialisation de la pensée symbolique,22 messo al servizio dell'esperienza individuale e soggettiva del singolo. Nel romanticismo quest'aspetto trova la sua massima espressione, portando alla realizzazione di una scrittura, nel senso attuale del termine, mentre prima esisteva solo un discorso orientato in senso teocentrico.

Nell'interpretazione di Bonnefoy, Leopardi, per molti aspetti, si pone in linea con i Romantici, in particolare con Keats (accostato al poeta di Recanati nella raccolta di traduzioni Keats et Leopardi) e Wordsworth, differenziandosene però per altri. Non cerca di trovare una sintonia tra sé e la natura, quanto invece di evadere da essa; in

\footnotetext{
18 Y. Bonnefoy, L'enseignement et l'exemple de Leopardi, Périgueux, William Blake \& Co, 2001, p. 10.

${ }^{19}$ Ibidem.

20 Ibidem.

${ }^{21}$ Ivi, p. 12

22 Ibidem.
} 
questa luce viene riletta dal poeta francese la stessa esperienza del 'naufragio' nell'Infinito: 'Il essaie de ne faire du néant de sa condition et de l'infini de son rêve qu'une seule grande expérience, qui est de se perdre dans l'abîme de l'incréé'. ${ }^{23}$ L'esperienza del naufragio de L'infinito viene assimilata a quella dei mistici distaccati da qualsiasi ricordo di sé e anche il messaggio della Ginestra in cui il poeta evoca il divario esistente tra il vuoto orgoglio del genere umano che si crede 'fine [...] al Tutto' e, di contro, la prospettiva decentrata, assunta in seguito all'indifferenza della natura, lo porta ad avere quell'intuizione del nulla che lo pone in linea con altri poeti come Mallarmé.

$\dot{E}$ a questo punto che Bonnefoy muove un'obiezione al letterato italiano riguardante proprio il contrasto tra la scoperta del pensiero nichilista e i sentimenti di Leopardi che non sono di distacco, ma di accusa appassionata alla natura crudele, valorizzando di contro soddisfazioni mediocri e ingannevoli quali il sogno, l'amore e soprattutto la noia definita 'il più sublime dei sentimenti umani':

En bref, la nature n'est pas pour Leopardi l'indifférence qui le recouvrira sans avoir rien su de lui, elle est le mal qui l'accable, qui l'oblige à désirer le non-être comme le refuge paradoxal de la réalité proprement humaine: et au moment même où il reconnaît le néant de cette dernière, c'est donc comme s'il en faisait un absolu cette fois encore, et restait ainsi prisonnier de la vieille illusion anthropocentrique. L'illimité que ce poète ressent dans le désir est une façon pour l'humain de se percevoir comme le lieu propre de l'infini, dans un monde de finitudes. C'est le signe qu'il est d'essence divine. ${ }^{24}$

Bonnefoy si rende anche conto che il pensiero di Leopardi, a causa della genericità di molti giudizi che enuncia, sul male, la natura e così via, ha favorito la sua ricezione in forma aneddotica, incentrata sulle vicende autobiografiche del letterato condizionato nei suoi giudizi dalla malattia e dalla solitudine e pertanto studiato esclusivamente come poeta. L'autore francese intende invece porre l'accento sul 'discours de Leopardi dans son œuvre', ${ }^{25}$ sulla proposta di un pensiero che se non è nuovo (in quanto già diversi pensatori europei avevano sostenuto tesi analoghe) diventa innovativo nella misura in cui trova il suo spazio nella poesia.

Il saggio prosegue poi con bellissime pagine di critica letteraria in cui Bonnefoy passa ad analizzare alcuni componimenti leopardiani, a cominciare dal Canto notturno di un pastore errante dell'Asia. Qui il discorso procede scegliendo simboli dal mondo esterno, in primis la luna, simbolo che se da una parte deve significare 'son néant et éclairer sa déréliction', ${ }^{26}$ dall'altra è anche una 'giovinetta immortal' alla quale si rivolge un pastore, confrontando il proprio destino col suo. In questo momento dialogico Bonnefoy vede non una riprova dell'indifferenza dell'astro, e quindi della natura, alle vicende umane, quanto invece il disvelamento di

un rapport au monde qui s'était empiégé dans l'idée de la matière, du non-sens éternel et universel, de la détresse obligée, n'a pas pour effacer le désir, serait-il surtout nostalgique, d'une relation interhumaine, et plus précisément d'homme à femme, qui éclairerait l'esprit, atténuerait l'inquiétude, et se prolongerait pour toute la durée d'une vie dans les interminati spazi de l'existence. ${ }^{27}$

La luna fanciulla è considerata una costante della poesia leopardiana: la ritroviamo in Alla luna come 'graziosa' e 'diletta', anche in questo caso confidente dell'io lirico;

\footnotetext{
23 Ivi, p. 14.

24 Ivi, p. 17.

${ }^{25} \mathrm{Ivi}$, p. 18.

${ }^{26} \mathrm{Ivi}$, p. 20.

27 Ivi, pp. 20-21.
} 
all'inizio della Sera del di di festa è 'queta', e poi ancora è 'cara' e 'benigna delle notti reina' nella Vita solitaria. Attraverso il tema selenico, insomma, affiorano, all'interno del discorso di denegazione del mondo, parole di adesione ad alcune forme del mondo stesso.

Inoltre, e su quest'aspetto Bonnefoy si sofferma per molte pagine, lungo la parte conclusiva del suo bel saggio, una specificità dei versi leopardiani è la loro musicalità che permette alla parola di aprirsi alla presenza del mondo stesso, proprio grazie a questa dinamica di musicalizzazione dell'enunciato lirico, materiato dal canto di Silvia, ma anche dal progetto di una pièce teatrale sulla regina di Francia Maria Antonietta, ipotizzando per lei 'une musique sans mots', per meglio esprimere il mistero della condizione mortale di cui la regina poteva essere l'emblema. Ma, in fondo, si evince dal proseguire del discorso di Bonnefoy, anche la poesia è in grado di dar voce a questi concetti, dal momento che le parole sono costituite di materia sonora. Ecco allora, con Leopardi, realizzarsi poèmes musicaux che assumono una valenza evocativa del ricordo, anzi della 'ricordanza', parola che reca in sé 'cor' e 'danza', il sentimento e il riferimento alla sfera musicale, cruciale nella rilettura critica di Orcel.

In modo sapiente e insieme con tono affabulatorio, Bonnefoy spiega insomma l'originale dialettica presente nel pensiero leopardiano tra materialismo e forza dell'illusione, dialettica in parte messa in luce da tanta critica leopardiana, ma qui riletta attraverso alcune parole chiave della poetica dell'autore francese che, cogliendo la correlazione apparentemente ossimorica tra il nulla e la musica, riesce a mostrare l'essenza stessa della scrittura leopardiana, ruotante intorno alla 'doppia postulazione' del nulla e dell'essere e portatrice di un forte messaggio di speranza, sia pur illusorio.

Se L'enseignement et l'exemple de Leopardi costituisce indubbiamente il principale punto di riferimento teorico su Leopardi scritto da Bonnefoy, occorre ricordare come, già negli anni precedenti, lo scrittore francese si fosse soffermato, nell'ambito di interventi pubblici, sul poeta di Recanati. Ne è un esempio lo scritto Pour introduire à Leopardi, testo della prolusione tenuta il 14 dicembre 1998 alla Sorbona in apertura del colloque su 'Leopardi philosophe et poète, 1798-1998. Presence et rayonnement de Leopardi dans le monde'. In questo discorso si trovano i nuclei ragionativi portanti della successiva riflessione di Bonnefoy, a cominciare dall'inquadramento storico col riferimento alla filosofia illuminista e il ritorno, con la poesia romantica, alla ricerca nella natura di un principio divino, espresso in vario modo da Lamartine, Vigny, Victor Hugo; d'altro canto si sviluppa un filone di pensiero che mira a vivere senza illusioni, come ben esemplificato da Mallarmé in Francia. Ma, nota Bonnefoy, già prima qualcuno in Italia aveva ripercorso un'esperienza indubbiamente simile.

Citando sempre l'esempio del Canto notturno, il critico-poeta mostra come Leopardi abbia additato all'umanità intera l'assenza totale di ordine nelle cose della natura, una luna fatta solo di materia, un cielo vuoto, dichiarando apertamente la sua esigenza di tradurre una poesia tanto bella, che è anzi una ri-traduzione, ma necessaria per far rinascere i versi nelle parole di un'epoca nuova:

Ce poème est si beau, autant que si éloquent, que je n'ai pas résisté au besoin de le traduire en français, il m'a semblé que notre langue en avait besoin, que sa lumière devait se répandre aussi dans nos mots: si bien qu'une traduction nouvelle ne serait pas de trop, malgré la qualité d'autres déjà existantes. ${ }^{28}$

${ }^{28} \mathrm{Ivi}$, p. 33. 
Ritroviamo anche il concetto della centralità della musicalità dei versi dei Canti a dispetto di un contenuto 'rude' e 'violent', di una 'pensée noire', rara in tutti i poeti europei, eccezion fatta per qualche strofe di Keats.

Bonnefoy coglie nella poesia di Leopardi un'enorme fiducia nel valore risanante della parola poetica ('c'est comme si la parole de Leopardi réparait le malheur du monde qu'elle avait pourtant dénoncé' $)^{29}$ come è ravvisabile in modo chiaro nell'Infinito. Secondo questa interpretazione, infatti, l'immensità che si lascia intravedere, al di là della siepe, non è che una metafora di ciò che svela la parola poetica. Si delinea cioè una dimensione simile a quella onirica in cui è come se le parole e i ritmi allineassero in modo armonico le relazioni tra le cose, in modo persino accogliente, come avviene in certi notturni o nell'immagine della luna, in tutto identificabile a una fanciulla che corrisponde a un desiderio d'amore. In altre pagine, più dure e prive di queste aperture gioiose, $i$ testi, privati del sogno, riflettono pienamente il néant della condizione umana; alcune pagine leopardiane sembrano allora precedere quelle di Samuel Beckett, tra i tanti esempi possibili.

Il poeta di Recanati è considerato un poeta moderno nel senso più pieno della parola ovvero uno di quei poeti che nella modernità 'ont su avoir l'intuition qui permet à la poésie de sortir de l'impasse où la pensée du non-être risque de la faire se perdre'. ${ }^{30}$ Come già Leopardi stesso aveva espresso chiaramente nel Discorso di un italiano intorno alla poesia romantica, la poesia ha allora il compito di mantenere in vita quelle illusioni secolari e quelle menzogne che ci permettono di vivere. E così, in conclusione, è sintetizzata la posizione di Bonnefoy (e il suo giudizio di valore) intorno a Leopardi nel suo scritto L'enseignement et l'exemple de Leopardi:

La grandeur de Leopardi: moins d'avoir été le premier des poètes vraiment lucides que de n'avoir pas, en cette lucidité même, méconnu l'évidence que celle-ci risque de faire oublier. D'un mot, n'avoir pas conclu du non-être de l'univers au non-sens du monde que nous a ouvert le langage. ${ }^{31}$

Secondo Bonnefoy il primo ad aver percepito le caratteristiche reali del mondo naturale è stato proprio Leopardi. Al sole da cui agli occhi di molti poeti emanavano $i$ raggi della luce divina, si è adesso sostituita la luna intesa come un freddo blocco di roccia, nonostante il suo volto luminoso. Ma la sua luce non è che un semplice aspetto del mondo fisico, diventa l' 'aboutissement dans l'esprit'32 di un processo cominciato due secoli prima con Galilei. L'uomo cessa allora di essere una creatura eletta da Dio, ma diventa un essere sofferente costretto a muoversi erraticamente, meravigliato della sua stessa sorte, come viene espresso nel Canto Notturno che lo scrittore francese definisce in questa prolusione 'un des plus beaux poèmes de l'Occident' 33 in cui si esprime insieme la miseria metafisica della condizione umana e l'emozione che pervade l'individuo quando ne prende coscienza. Nuova, secondo il critico francese, è anche la forma del componimento, libera da costrizioni prosodiche e ricca di alcune caratteristiche che hanno spinto il lettore Yves a divenire traduttore di quei bei versi e a seguire un impulso simile a quello provato dallo stesso Leopardi quando, trovandosi di fronte alle bellezze delle opere greco-latine, avvertiva 'un desiderio ardentissimo di tradurre'. Ecco allora che, percorrendo questa trafila di senso, le motivazioni di Bonnefoy assumono la forma del desiderio: 'Et j'ai désiré traduire ce poème, parce

\footnotetext{
29 Ivi, p. 35.

30 Ivi, p. 38.

31 Yves Bonnefoy, L'enseignement et l'exemple de Leopardi, cit., p. 39. Corsivi nel testo.

32 Ivi, p. 44.

33 Ivi, p. 45.
} 
qu'il est si véridique qu'il faut absolument en intérioriser l'intuition dans la langue que l'on parle, et dont il faut dissiper les rêves'. ${ }^{34}$

Commentando l'incipit del Canto Notturno, Bonnefoy spiega la sua felice formulazione critica di 'seconde intuition', per molti aspetti assimilabile alla 'doppia postulazione' di cui si era servito per mostrare il dualismo caratterizzante la nazione italiana:

Qu'est-ce que cette seconde intuition, dont je crédite Leopardi? Et bien, c'est de percevoir que si le fait humain ne repose sur aucun substrat ontologique, aucun étayage au dehors qu'il puisse dire réel et non illusoire, l'être parlant n'en a pas moins mis en place, par l'institution du langage, un lieu de représentations, de significations, de valeurs où chaque être qui parle est présent à la présence de tous les autres d'une façon si immédiate et accaparante, si cohérente aussi et indéfiniment praticable, qu'il n'y a pas de raison pour ne pas tenir ce champ d'existence pour une réalité en soi, à laquelle il sera loisible de conférer une valeur absolue. Dans cet espace existentiel, la personne n'est certes pas illusoire, puisqu'elle en est comme telle la raison d' être. Et à la place ses significations énigmatiques qui s'enchevêtrent pour rien dans notre rapport au monde physique, un sens également bien réel prend forme comme reconnaissance de nos besoins dans la vie. Une réalité, proprement humaine, un lieu où l'être est, parce que nous décidons qu'il sera. Et d'évidence une tâche, pour la parole: élaborer ce sens, le nourrir des aspects du monde auxquels nous trouvons du prix. Faire, en somme, de la lucidité radicale, la clef d'un être-au-monde enfin proche du corps, enfin libre. ${ }^{35}$

Analogamente, nella Sera del di di festa, alla meditazione scorata sulle età passate e sul destino dell'umanità, basta un tenue elemento come la voce di un passante per strada nel buio della notte per introdurre d'un tratto un elemento nuovo, una présence, un canto che 'se fait le chiffre du lieu et du devoir de la poésie, dont il faut attendre qu'elle enjambe le désarroi de notre heure présente post-moderne pour recommencer sur des bases enfin solides le mouvement de l'esprit'. ${ }^{36}$

Attraverso queste due poesie citate a titolo esemplificativo, infatti, appare chiaro, secondo Bonnefoy, in cosa consista il nostro debito verso Leopardi: 'une clarification de la tâche poétique', in modo analogo a quella che Rimbaud, ${ }^{37}$ in Francia, avrebbe formulato in seguito. Il messaggio leopardiano è di grande attualità e deve essere ascoltato e continuamente richiamato alla memoria in un momento di grande difficoltà e di continue insidie per lo spirito: un messaggio da rilanciare con forza proprio per la sua 'naïveté fondatrice'.

\section{Orcel traduttore e i Chants leopardiani}

Alla rilettura 'luminosa' e poietica dell'Italia di Bonnefoy, operata attraverso il continuo richiamo allo splendore del patrimonio artistico-letterario italiano, riteniamo significativo accostare l'Italie obscure di Michel Orcel che, alla consueta immagine della nostra penisola come 'jardin des lettres et des arts, paradis voluptueux du

\footnotetext{
34 Ibidem.

35 Ivi, pp. 46-47.

36 Ivi, p. 47.

37 Anche Rimbaud è da annoverare tra gli autori cari a Bonnefoy dal suo Rimbaud par lui-même del 1961 al recente Notre besoin de Rimbaud (Paris, Seuil, 2009). Di quest'autore il letterato francese loda la sorprendente modernità, declinata coi toni del maledettismo tra vita difficile, morte precoce e gloria duratura. Si ricordi, infine, per inciso che anche in Rimbaud è molto importante e noto il tema del naufragio, presente, con accenti diversi, in Mallarmé e naturalmente in Giacomo Leopardi. Cfr. Carlo Ossola, 'Rimbaud, eterno naufragio', in: Domenica. Il sole 24 ore, 101 (12 aprile 2009), p. 31.
} 
chant $^{38}$ oppone la complessità del pensiero moderno maturato dal Rinascimento in poi, a partire da Ariosto e dalla malinconia tassiana, per poi concretizzarsi in quel 'prodigieux travail de négation' di Leopardi. Si delinea cioè una prospettiva per molti versi capovolta rispetto a quella di Bonnefoy, più cupa e problematica come si cercherà di precisare, ma al tempo stesso affine, soprattutto nella proposta di pagine critiche elaborate in seguito a un rigoroso e appassionato percorso traduttivo.

Nel saggio introduttivo Contre Stendhal (pour ainsi dire) Orcel sottolinea come si sia creata una nuova e perturbante relazione del soggetto con il suo ambiente geografico e sociale, a partire dal declino rinascimentale, dalle vertigini dell'età barocca sino all'inquietudine metafisica del preromanticismo. Per spiegare questo lato oscuro dell'Italia lo scrittore si rifà all'importante tema dell'illusione e alla sua successiva 'défaillance'. ${ }^{39}$

Notevole è l'impegno divulgativo profuso in Francia da Orcel come traduttore di Leopardi: se molte sono le riflessioni di Orcel sul tradurre, e in particolar modo sulla laboriosa traduzione del capolavoro tassiano, non disponiamo però di altrettante indicazioni specifiche sull'impegnativo lavoro di traduzione complessivo dei Canti di Leopardi, se non per le note del traduttore, ${ }^{40}$ poste come premessa dell'edizione completa e riveduta dei Canti, già pubblicati nel 1987 per i tipi di La Dogana (Genève) col titolo di Poèmes et fragments. Si legga, a scopo esemplificativo, la traduzione dell'Infinito:

\author{
L'Infini \\ Toujours tendre me fut ce solitaire mont, \\ Et cette haie qui, de tout bord ou presque, \\ Dérobe aux yeux le lointain horizon. \\ Mais couché là et regardant, des espaces \\ Sans limites au-delà d'elle, de surhumains \\ Silences, un calme on ne peut plus profond \\ Je forme en mon esprit, où peu s'en faut \\ Que le cœur ne défaille. Et comme j'ois le vent \\ Bruire parmi les feuilles, cet \\ Infini silence-là et cette voix, \\ Je les compare: et l'éternel, il me souvient, \\ Et les mortes saisons, et la présente \\ Et vive, et son chant. Ainsi par cette \\ Immensité ma pensée s'engloutit: \\ Et dans ces eaux il m'est doux de sombrer.
}

Una prima variazione che emerge in questa ri-traduzione del celebre componimento leopardiano, uno dei più antologizzati, in Italia e all'estero, si ha nella resa dell'aggettivo 'dolce' con 'tendre', scelta dissimile da quella di tutti gli altri traduttori dei Canti da noi presi in esame e che rende bene il senso metaforico del qualitativo italiano, accentuandone, forse, col richiamo alla tenerezza, la componente affettiva, di vicinanza anche emotiva col luogo evocato. Analogamente 'ermo colle' è reso con 'solitaire mont' che verrà fatto rimare con 'horizon'.

\footnotetext{
${ }^{38}$ M. Orcel, Italie obscure, cit., quarta di copertina. Mario Andrea Rigoni si è soffermato sui lavori di italianistica di Michel Orcel nel suo 'Michel Orcel e l'individualità italiana', in: Lettere italiane, LIII, 1 (2001), pp. 96-101.

39 Ivi, p. 8.

${ }^{40} \mathrm{Cfr}$. M. Orcel, Notes sur la traduction in Giacomo Leopardi, Chants/Canti, traduction et présentation par Michel Orcel, préface par Mario Fusco, Paris, Flammarion, 2005, p. 21. Tutte le citazioni successive di queste Notes fanno riferimento a questa pagina. Analogamente le traduzioni da Orcel sono tratte da questa edizione.
} 
Tutti i traduttori si trovano in difficoltà nel rendere 'tanta parte dell'ultimo orizzonte', qui si sottolinea che l'esclusione riguarda "quasi tutta" la visuale ('tout... ou presque'), con la scelta, già di Char, di significare l'esclusione col verbo 'dérobe'. Il guardo viene reso con 'yeux' e l' 'ultimo orizzonte' diventa 'lointain'.

La dittologia verbale 'sedendo e mirando' prevede un costrutto simile (laddove Char, ad esempio, aveva esplicitato diversamente i due verbi) con la sostituzione del verbo 'sedersi' a un altro termine che evoca invece il coricarsi, favorendo la contemplazione da una posizione distesa. Dei traduttori presi in esame solo Jaccottet mantiene la forma del doppio gerundivo. Correttamente Orcel individua il valore del 'mi fingo' leopardiano esplicitandolo con una perifrasi 'je forme en mon esprit', rendendo bene gli oggetti di questa vista con espressioni che danno l'idea del limite e del suo contrario ('sans limites', 'surhumains' e la perifrasi 'on ne peut plus profond' per il superlativo 'profondissima'). In alcuni casi il traduttore si avvale di forme desuete come 'j'ois'. La resa degli indicatori spaziali ('questo', 'quello' ecc.) costituisce una difficoltà per i traduttori dal francese in cui l'unico dimostrativo 'ce' può essere rafforzato da '-ci' o '-là' come avviene in questo caso ('couché là', 'silencelà') senza però ottenere la dialettica di vicinanza o lontananza dall'infinito presente nel testo leopardiano. 'Il suon di lei' è reso con 'son chant' e, leggendo lo studio critico di Orcel, Il suono dell'Infinito, si può capire come per il traduttore il richiamo "musicale" possa costituire il centro nevralgico del componimento.

La conclusione con la sequenza mirabile del perdersi nell'infinito è resa enfatizzando l'effetto di sprofondamento del pensiero già anticipata nel verso 8 della traduzione (con 'défaille' riferito al cuore) e negli ultimi versi con la traduzione del verbo 'annegare' con 's'engloutit' e con 'sombrer' nelle 'eaux' che rappresenta certo una variante al naufragio nel mare.

Nell'insieme emerge comunque che Orcel, rispetto agli altri poeti-traduttori novecenteschi presi in esame (Bonnefoy, Jaccottet, Char...) si mantiene più prossimo al testo di partenza ${ }^{41}$ similmente alla scelta già attuata dal poeta-traduttore Philippe Jaccottet, il quale però, tende ad assumere una posizione per lo più cibliste al contrario di Orcel che talvolta, nei Canti, introduce, a differenza di altri traduttori, dei termini arcaici o 'peregrini', preservando così l'alterità del testo tradotto, servendosi di alcuni effetti stranianti che coinvolgono l'originale.

Questa poesia è stata oggetto anche dell'attenzione critica di Orcel, autore del denso saggio /l suono dell'infinito. Sicuramente non consueta è la prospettiva adottata da Orcel per analizzare il celebre componimento. Egli osserva innanzitutto come ci si trovi di fronte alla sola poesia dei Canti dove non compaia il tema, tipicamente leopardiano, del dolore e che sembra invece sorto 'da un altrove della parola' 42 emerso in modo nuovo ed enigmatico al punto da aver suscitato le interpretazioni più varie, dall'esperienza religiosa al nichilismo, dal processo ironico alla regressione allo stadio prenatale. Orcel si propone allora, come novello Candide, di rileggere il testo da una prospettiva ingenua (fittiziamente aggiungeremmo noi), individuando il punto focale dell'idillio nella manifestazione del vento, elemento, secondo lo studioso, non

\footnotetext{
41 Secondo Ariane Luthi ('Bonnefoy traducteur de Leopardi: (re)traduire l'Infinito', in: Revue de BellesLettres, CxxIX, 3-4 (2005), p. 102) nel caso di Orcel si potrebbe parlare di una 'version interlinéaire' in quanto "ainsi la ponctuation est-elle identique à celle du poème italien, et il ne reproduit pas seulement les huit “et", mais les huit conjonctions se trouvent aussi au même endroit que chez Leopardi'. Simile è anche il parere di Mathilde Vischer, la quale così scrive: 'La qualité de la traduction de Jaccottet est d'offrir un poème équilibré en français, tandis que la traduction d'Orcel, si elle ne donne pas un poème aussi riche du point du vue sonore, suit de manière plus précise "la lettre" du texte, en respectant les nuances de rythme et de sens' (M. Vischer Mourtzakis, Philippe Jaccottet traducteur et poète: une esthétique de l'effacement, Lausanne, Centre de Traduction Littéraire de Lausanne, 2003, p. 53).

42 Si cita da M. Orcel, 'Il suono dell'infinito', in: M. Santoro (a cura di), Leopardi nella critica internazionale, Napoli, Federico \& Ardia, 1989, pp. 125-153.
} 
sufficientemente approfondito nei pur numerosi studi critici su questo componimento. Dopo un dettagliato procedere argomentativo emerge che la vera chiave di volta del discorso è data dal significato del verbo 'comparare' nel verso 11 del testo leopardiano da non intendersi come 'paragonare', bensì come 'accoppiare', 'unire', ovvero percependo il vento in uno spazio indeterminato, il poeta "va comparando", cioè unisce la 'voce' all' 'infinito silenzio'. Avviene quindi una:

trasmutazione, di cui il soggetto non è più la causa [...]: la voce del vento trasmuta l'infinito spaziale in 'eterno', vale a dire nel passato più remoto, nell'origine astratta del tempo. [...] E quindi si spiega l'annientamento felice nell'immensità, di cui non poteva logicamente rendere conto l'interpretazione tradizionale. È nell'immensità stessa di questa voce ('questa immensità'), nella dismisura di questo suono primordiale, che il pensiero può abolirsi regredendo verso l'Origine in una percezione del tempo insieme sacrale e primitiva. ${ }^{43}$

Da queste osservazioni tutta la poesia viene riletta alla luce dell'originario, del primordiale, operante entro la duplice poetica dell'idillio: quella del 'vago' e dell'indefinito e quella che Orcel chiama 'del primitivo'. La poesia viene quindi analizzata 'come luogo di una riattivazione dell'immaginazione originaria' insieme infantile e primitiva che si esprime metaforicamente. Se poi si riflette che i principali "prodotti" dell'immaginazione primitiva e infantile sono le illusioni è possibile ulteriormente rendersi conto della portata dell'esperienza centrale, quella della Voce del vento.

L'ultimo aspetto a essere ampiamente analizzato in questo saggio è quello che associa i ricordi infantili a reminiscenze auditive o musicali. Orcel ricostruisce allora con acutezza una sorta di diario 'acustico' rintracciabile all'interno della produzione leopardiana, che motiva meglio la dialettica individuata nell'Infinito ('voce - eterno / canto - principio del mondo). ${ }^{44}$ La musica, come espresso, ad esempio, in molti passi zibaldoniani permette un 'ritorno all'antico' e il suono viene inteso come fenomeno originario, 'materia della musica' stessa, intesa alla maniera di Wackenroder, come arte asemantica in quanto:

Le altre arti imitano ed esprimono la natura da cui si trae il sentimento, ma la musica non imita e non esprime che lo stesso sentimento in persona, ch'ella trae da se stessa e non dalla natura, e così l'uditore. [...] La parola nella poesia ec. non ha tanta forza d'esprimere il vago e l'infinito del sentimento se non applicandosi a degli oggetti, e perciò producendo un'impressione sempre secondaria e meno immediata, perché la parola, come i segni e le immagini della pittura e scultura, hanno una significazione determinata e finita. (Zib. 79-80).

La musica 'arte dell'infinito del sentimento', ${ }^{45}$ dunque, che ha la capacità di "immergere" l'ascoltatore nell'abisso dell'indeterminazione del senso: è quanto proposto nell'Infinito, come confermato anche da altri versi dei Canti. ${ }^{46}$

Un'insistenza verso tonalità che virano sempre verso il bianco, un chiarore che è quello nivale, ma anche rimando alla vecchiaia e quindi alla morte, percorre tutta la traduzione del Canto notturno, intitolata Chant nocturne d'un berger errant de l'Asie.

\footnotetext{
${ }^{43}$ Ivi, p. 130.

44 Ivi, p. 138.

45 Ivi, p. 151. Corsivi nel testo.

46 Orcel (ivi, p. 152, nota 35) fa opportunamente riferimento a versi tratti da Aspasia (vv. 33-37 e 67-70) e soprattutto ai versi 39-49 di Sopra il ritratto di una bella donna: 'Desiderii infiniti / e visioni altere / crea nel vago pensiere, / per natural virtù, dotto concento; / onde per mar delizioso, arcano / erra lo spirto umano, / quasi come a diporto / ardito notator per l'Oceano: / ma se un discorde accento / fere l'orecchio, in nulla / torna quel paradiso in un momento'. Quest'ultima citazione, secondo lo studioso francese, avrebbe dovuto da tempo indirizzare i commentatori leopardiani verso un'interpretazione dell'Infinito come esperienza musicale.
} 
Per tradurre 'in sul primo albore' (v. 11), ad esempio, Orcel sceglie l'espressione 'aux premières blancheurs' e ancora il 'vecchierel bianco, infermo' del verso 21 è un 'vieillard fragile et blanc'; per rendere l'ultimo appellativo rivolto alla luna 'candida luna' (v.138) si ha il ricorso al medesimo aggettivo ('blanche Lune'). Questi riferimenti che, fedeli al testo leopardiano, privilegiano comunque questo preciso tratto cromatico (sulla cui semantica si è accennato nei discorsi sulla neve) precisano lo sfondo invernale, di freddo e ghiacci (e naturalmente 'glace' è pure fittamente ricorrente in questi versi), proposto in più luoghi del Canto, allineandosi, al tempo stesso, alle scelte di altri traduttori leopardiani.

Per il resto, di questa traduzione, si segnala la scelta di rendere 'tedio' con 'ennui' e si riporta il verso finale del testo 'jour funèbre est pour qui naît le jour natal' che traduce il celebre 'è funesto a chi nasce il dì natale'. Si verifica poi un inevitabile impoverimento del testo di partenza, soprattutto nella resa degli effetti fonici che conferiscono una particolare semanticità alla rima in -ale posta alla fine di ogni stanza e non riprodotta nelle traduzioni francesi. Da questi esempi emergono molte peculiarità di Orcel traduttore, attento al rispetto della lettera e insieme in grado di trovare soluzioni originali per il testo di arrivo. ${ }^{47}$ Il suo volume dei Canti è arricchito inoltre da un'introduzione e da note di commento alle singole poesie. Dopo la prefazione di Mario Fusco, che ha la funzione precipua di introdurre Leopardi al pubblico francese, vi è il saggio di Orcel dal titolo Leopardi et le procès des formes.

Riprendendo alcune linee interpretative già suggerite in contributi precedenti, ${ }^{48}$ lo studioso si sofferma sul significato del titolo Canti, insistendo molto sull'elemento musicale presente in questa raccolta. Dal Risorgimento in cui sono evidenti le vestigia metriche e linguistiche dello stile metastasiano e quindi dei libretti d'opera, alla 'réactivation massive de la forme canzone', ${ }^{49}$ prevalente dal punto di vista quantitativo, dall'uso della canzonetta e della terza rima. Le forme classiche sono innovate da Leopardi dall'interno, sia dal punto di vista metrico quanto soprattutto da quello contenutistico aprendo la strada, ad esempio, al lessico dell'indefinito.

Spunti critici abbastanza divergenti rispetto al panorama di studi consueto si trovano anche in Ombres du plaisir. Entretien avec Michel Orcel, un'intervista pubblicata nel numero monografico dedicato a Leopardi della rivista 'Europe'. Orcel, rispondendo alle domande di Jean-Baptiste Para, comincia col ridimensionare la leggendaria precocità leopardiana, frutto, a suo giudizio di una certa agiografia leopardiana, ritenendo il piccolo Giacomo certo intellettualmente molto dotato, ma insistendo molto sull'iniziale erudizione più che sulla sua precoce genialità, come poteva invece averla avuta Mozart o Rimbaud. Dal punto di vista specificamente poetico, infatti, il primo importante testo teorico, il Discorso di un italiano sulla poesia romantica si data al 1818, quando Leopardi aveva venti anni e il primo vero capolavoro poetico è L'infinito del 1819.

Orcel si propone di sfatare inoltre l'idea dei traumi infantili subiti dal piccolo Giacomo di cui suppone invece un'infanzia complessivamente felice e gioiosa. E anche quando, intorno ai dodici anni, si trova già in preda a ossessioni religiose e a una smania

\footnotetext{
${ }^{47}$ M.A. Rigoni, nel suo articolo 'Michel Orcel e l'individualità italiana', cit., p. 98, nota come 'la concreta sensibilità poetica e verbale di Orcel [...] riesce talvolta a conservare nella traduzione persino ciò che ordinariamente non è traducibile da una lingua in un'altra, ossia il ritmo, balza agli occhi di chiunque legga la sua versione dei Canti di Leopardi: nel tentativo felice di prendere il passo dell'originale, essa può rassomigliare sotto certi aspetti alla bella versione che Pierre Klossowski ha dato dell'Eneide'.

${ }^{48}$ Cfr. almeno M. Orcel, Langue mortelle, Paris, L'Alphée, 1983 (soprattutto capitolo III, 'Le son de l'Infini', pp. 124 e segg.).

49 Id., Leopardi et le procès de formes, in G. Leopardi, Chants/Canti, cit., p. 13.
} 
quasi compulsiva di sapere, è capace di scrivere, per l'Epifania, una lettera impertinente e scherzosa a un amico del padre.

Un rammarico di Orcel è inoltre costituito dal fatto che nonostante sia stato compiuto qualche tentativo di offrire un contributo di impostazione analitica su Leopardi, ${ }^{50}$ non esiste ancora un vero e proprio studio psicocritico. La malinconia è intesa dallo studioso 'du côté du vrai' che gli permette di far sì che la sua soggettività si ponga come prototipo della Décadence baudelairiana e di istanze più universali. In questo scenario l'idillio inteso secondo l'etimo greco come 'petite image' non è più possibile entro un'esistenza priva di immagini. A questo punto intervengono semmai le illusioni e quindi la speranza, che in Leopardi sono saldamente intrecciate sul piano speculativo col desiderio e l'amore di sé.

Nella sua opera di diffusione dell'opera leopardiana Orcel si è peraltro dedicato anche alla prosa, mostrando il 'petit théâtre philosophique' delle Operette morali nel volume intitolato Six petites pièces philosophiques in cui traduce sei operette. ${ }^{51}$ Ben articolata appare la prefazione in cui Orcel presenta quest'importante opera leopardiana, scandita da alcuni punti di approfondimento: dalla scomparsa progressiva delle illusioni, espressa attraverso il ricorrente topos del deserto all'equivalenza stabilita tra Désire malheur, sino al considerare il Dialogo della Natura e di un Islandese come il romanzo autobiografico che Leopardi non riusci mai a scrivere. $\mathrm{E}$ ancora ben approfondite sono le pagine dedicate al 'soleil noir' della Morte nel Coro dei morti, considerato da Orcel, come si diceva, una delle poesie più belle e inquietanti non solo del Recanatese, ma di tutto il secolo e alla composizione di 'poésie en prose' in alcuni passaggi delle operette.

Acuta è anche la sua lettura dell'Elogio degli uccelli solo erroneamente considerate un intermezzo euforico entro il quadro desertico delle Operette. Orcel dimostra invece, con puntualità di rimandi, che l'Elogio è l'esempio di una 'création fictionelle dans le désert des illusions' ottenuta attraverso la forma più sofisticata, cioè l'ironia. Anzi, precisamente, attraverso la parodia esatta e raffinata dell'encomio, dell'elogio greco, come avviene nell'Elogio della mosca di Luciano che è considerato una fonte delle Operette.

In un paio di casi anche Orcel fa riferimento al motivo glaciale associato a Leopardi: circolarmente all'inizio e alla fine della sua penetrante prefazione alle Operette. Dapprima precisando la particolare forma di classicismo, anzi di 'hellénisme' in Leopardi in termini di 'glaciale componction' e alla fine, a proposito del Cantico del Gallo silvestre, con l'evocazione di un cosmo 'froid' tra un 'silenzio nudo' e una 'pace profonda'. Tracciando, infine, le conclusioni del suo lavoro, il critico-traduttore francese individua in questo fondamentale lavoro di Leopardi un saggio di filosofia morale orientata, sul piano collettivo, verso la solidarietà (senza però alcuna speranza) contro il male dell'esistenza incarnato dalla Natura indifferente e distruttrice, e sul piano individuale, verso una quiete (senza illusione) tendente all'atarassia, all'annichilimento del desiderio. ${ }^{52}$

Per poi cosi concludere parlando a proposito dei vari testi che compongono l'opera:

Toutes ne sont pas également bouleversantes, mais le plupart réussissent ce tour de force: animer en quelques pages des figures allégoriques ou enfantines, voire infantiles, dans une mise

${ }^{50}$ Cfr. G. Amoretti, Poesia e psicanalisi: Foscolo e Leopardi, Milano, Garzanti, 1979 e soprattutto E. Gioanola, Leopardi, la malinconia, Milano, Jaca Book, 1995.

51 Si fa riferimento a G. Leopardi, Six petites pièces philosophiques, choisies, présentées et traduites de l'italien par M. Orcel, Cognac, Le temps qu'il fait, 2009.

${ }^{52}$ Cfr. M. Orcel, Preface a Giacomo Leopardi, Six petites pièces philosophiques, cit., p. 18. 
à feu de la Raison comme puissance de destruction, sans que cette opération puisse jamais être isolée de son travail littéraire - linguistique et rhétorique -, où le capital séculaire se dépose pour former une image impitoyable de la modernité: le classicisme comme instrument de subversion. ${ }^{53}$

Dall'accostamento tra Bonnefoy e Orcel si delinea una particolare visione della penisola italiana entre-deux, bifronte, che guarda con ammirazione e acume critico all'Italia monumentale come a quella narrativa, contemperando questi aspetti con una visione lucida e disincantata, non dimentica dei suoi aspetti più contraddittori. Risulta inoltre efficace il confronto tra le diverse poetiche traduttorie dei due autori, soprattutto per il comune caso di Leopardi: più libere e target-oriented le versioni di Bonnefoy; maggiormente aderenti al testo e caratterizzate da notevole acribia quelle di Orcel. Il poeta di Tours si pone comunque come traduttore "occasionale" del Recanatese, selezionando il corpus da tradurre sulla base del suo gusto personale e di una profonda consentaneità del sentire, concedendosi spesso delle variazioni che sono veri e propri intarsi di alta poeticità. Orcel si dedica invece a un lavoro sistematico di traduzione dell'intera silloge dei Canti, prevalentemente source-oriented, realizzando un prodotto letterario di qualità che è divenuto oggi un punto di riferimento per $\mathrm{i}$ lettori e studiosi francesi di Leopardi, per il rigore e la sua capillare diffusione in Francia presso biblioteche e librerie.

La biplanarità di approccio che si è seguita nella stesura di questo contributo ha permesso di evidenziare come da due percorsi traduttori ben diversi ed egualmente originali e sapienti si generi un fecondo terreno comune di riflessioni critiche che porta Bonnefoy a rileggere, secondo la semantica della speranza, alcune poesie leopardiane come il Canto notturno di un pastore errante dell'Asia; dal canto suo Orcel critico interpreta L'Infinito e altri componimenti dei Canti secondo una prospettiva musicale, individuando al tempo stesso l'appartenenza di Ariosto-Tasso-Leopardi a una medesima e continuistica linea letteraria, caratterizzata dalla 'défaillance de l'illusion', fondamentale per il processo di formazione della coscienza moderna italiana dal Rinascimento al Romanticismo.

\section{Parole chiave}

Bonnefoy, Orcel, Italia, Leopardi, traduzioni

Novella Primo è dottore di ricerca sia in Italianistica (2002) che in Francesistica (2012), attualmente titolare di un assegno di ricerca in Letterature Comparate presso il Dipartimento di Scienze Umanistiche dell'Università di Catania. Ha conseguito l'abilitazione scientifica nazionale (ASN) alla seconda fascia della docenza universitaria nei settori concorsuali 10/F1 "Letteratura italiana, critica letteraria e letterature comparate" e 10/F2 "Letteratura italiana contemporanea".

Si è occupata in sede critica di Giacomo Leopardi (su cui ha pubblicato tre studi monografici), Primo Levi, Quasimodo, Jaccottet e Bonnefoy. Altri contributi sono stati dedicati a questioni di traduttologia nonché all'opera della scrittrice-pittrice Lalla Romano, studiata secondo una prospettiva di comparazione con l'Ecocriticism, la medicina narrativa e la fotografia.

\footnotetext{
53 Ivi, p. 19.
} 
Dipartimento di Scienze Umanistiche

Università degli Studi di Catania

Monastero dei Benedettini, Piazza Dante 32, studio n. 258

Catania (Italia)

primonove@gmail.com

\section{SUMMARY}

\section{Italie obscure or Une terre pour les images?}

The Italian Art and Literature of Bonnefoy and Orcel

Within the very wide panorama of contemporary French literature, Yves Bonnefoy (1923-2016) and Michel Orcel (born in Marseille in 1952) are two paradigmatic examples of the multi-faceted interest beyond the Alps towards Italy and its culture, evident in both writers as regards to essay writing, translation and the consequent metatraductive reflections, as well as in their creative productions. Despite the unavoidable differences in education and poetics, both scholars are comparable due to the common interest in the same Italian men of letters, for example Ariosto whose Orlando Furioso was translated by Orcel and finely interpreted by Bonnefoy - and particularly Leopardi, of whom both French authors have offered brilliant poetic translations and original essays. Considering some of their critical contributions to Italian art and literature (including Orcel's, Italie obscure, 2001 and Bonnefoy's, Une terre pour les images, 2005), the essay investigates the unique characteristics of the Italian landscape, on a natural and artistic level, which emerge from the writings of the two French authors so far examined. 